\title{
Bifurcation and Post-Bifurcation of an Inflated and Extended Residually-Stressed Circular Cylindrical Tube with Application to Aneurysms Initiation and Propagation in Arterial Wall Tissue
}

\author{
H. Dehghani ${ }^{a}$, D. Desena-Galarza ${ }^{\text {a }}$, N.K. Jha ${ }^{\mathrm{b}}$, J. Reinoso ${ }^{\mathrm{c}}$, J. Merodio $^{\text {a }}$ \\ ${ }^{a}$ Department of Continuum Mechanics and Structures, Escuela de Caminos, Canales y Puertos, Universidad Politécnica de Madrid, 28040 Madrid, Spain \\ ${ }^{\mathrm{b}}$ Hari Shankar Singhania Elastomer and Tyre Research Institute, JK Tyre, Mysore, India \\ ${ }^{\mathrm{c}}$ Grupo de Elasticidad y Resistencia de Materiales, School of Engineering. Universidad de Sevilla, Camino de los Descubrimientos $s / n, 41092$ Seville, Spain
}

\section{Keywords:}

Residual stress

Bending bifurcation

Bulging bifurcation

Nonlinear elasticity

Artery

Aneurysms

\begin{abstract}
A B S T R A C T
A numerical procedure to analyze bifurcation and post-bifurcation of a finite deformation boundary-value problem for a residually-stressed elastic body is studied. In particular, the problem is the combined extension and inflation of a circular cylindrical tube subject to radial and circumferential residual stresses. The material model, given by a residual-stress dependent nonlinear elastic constitutive law in terms of invariants, is implemented in a finite element code. A numerical procedure to analyze the bifurcation and post-bifurcation of the finite deformation boundary-value problem at hand is developed based on the modified Riks method. The dependence of bifurcation and post-bifurcation behavior of tubes under the loading at hand on residual stresses is shown and compared with results when there is no residual stress. The finite deformation boundary-value problem is described mainly in terms of the inflation pressure, as well as the axial and azimuthal stretches of the tube. The dependence of these quantities on bifurcation is illustrated graphically for different values of the parameters (in dimensionless form) involved, in particular, the strength of the residual stress. It is found that bulging bifurcation is expected for sufficiently large values of the axial stretch. On the other hand, for small values of the axial stretch (close to the non-extended configuration), the onset of bifurcation is found to be the bending mode. Furthermore, for the latter case in subsequent motion, i.e. post-bifurcation, it is shown that bending triggers bulging as opposed to the situation in which bending is not allowed and the onset of bifurcation is associated with bulging. In addition, the bulge, or the abnormal enlargement, that is formed during post-bifurcation after the onset of bending bifurcation appears on one side of the tube showing an irregular shape which is consistent with the development of abdominal aortic aneurysms (AAA).
\end{abstract}

\section{Introduction}

Bifurcation and post-bifurcation of different instability modes in thinwalled as well as thick-walled cylinders subject to several bound-ary conditions in the context of large deformation stability theory have attracted the attention of many researchers in the last few years (see Refs. [1-12]). An important application of this analysis is its relation with aneurysms formation and propagation in arterial wall tissue (see Refs. [1-4,6-11], for instance, and references therein). Many factors are involved in the formation and propagation of this pathology. These include among others geometry, nonhomogeneous material, anisotropy, growth, remodeling, age, etc. In addition, the evolution and forma- tion of an aneurysm is certainly based on biochemical processes. But a previous step to undertake that analysis is to understand the mechanical modulation of this process. We dedicate our effort to this aspect dealing with residual stress. Indeed, arteries are subjected to significant mechanical stresses that play important roles in regulating vascular cell biology, vascular function, and pathological development of vascular diseases (see Refs. [15-17]).

Bifurcation analysis involves, from a mathematical point of view, a complicated and rigorous machinery. Analytical solutions provide great insight into the physical interpretation of the problem at hand. Nevertheless, analytical approaches deal with specific material models, loading conditions and a perfect geometry and are only practical for 
relatively simple cases. It is important to supply alternatives to these difficult formulations. Here we approach this problem using the finite element method which is nowadays a basic tool in engineering as well as pure and applied sciences. A numerical method that can be used in these cases is the modified Riks method. This method considers the arc length of the load-displacement path as the constraint equation. That is the reason to also denote this method as the arc-length method. For the purpose of this communication, we specifically focus on the finite element code Abaqus/Standard which has implemented this procedure. Whence, an area of emphasis within this work following previous ones such as $[4,5,12]$, for instance, is also the development and use of numerical procedures to solve such bifurcation and post-bifurcation problems. This framework opens new possibilities and perspectives in both scientific and professional practice. It will clearly furnish an important source of expansion and progress to engineering analysis and design.

In recent years, a big effort has been made to investigate boundary value problems in the context of stability theory to address the stability of solutions of differential equations under small perturbations of initial conditions. This involves a variety of different geometries and loading conditions for different materials including arteries (see $[3,8])$. To model the evolution of aortic aneurysms the analysis needs to account for some thickness of the artery, which opens the possibility to include residual stresses (see Refs. [13,14]). Residual stresses are produced during the process of growth, development, and remodeling. They have an important influence on the mechanical behavior of arteries and hence may affect aneurysm formation and propagation. An aortic aneurysm is an abnormal enlargement or bulging of the wall of the aorta. An aneurysm can occur anywhere in the vascular tree. The bulge or ballooning is defined as a fusiform one when it is uniform in shape, appearing equally along an extended section and edges of the aorta. On the other hand, saccular aneurysms are related to a small, lop-sided blister on one side of the aorta. In the former case, and due to the symmetry of the geometrical solution, the bulging mode of bifurcation is exploited. The context of aneurysm formation with cylindrical geometry in cardiovascular diseases seems an appropriate framework for such aneurysms. From an analytical point of view, Fu and co-workers have made the most important contributions obtaining a bifurcation condition for localized bulging [11]. This localized bulging solution makes contact with a previous analysis that dealt with sinusoidal solutions when infinitely long tubes or tubes whose length is much greater than its radius [3] are considered. From a numerical point of view, Merodio and co-workers have analyzed these instabilities in a series of papers with the goal also to promote this (nonlinear) bifurcation analysis in engineering applications. In particular, the finite element method seems the appropriate tool to study post-bifurcation analysis [4] as well as to guide and validate analytical formulations [5].

The aforementioned papers dealing with bulging did not include residual stresses and we dedicate our effort to this endeavor. The residual stress formulation at hand can be reviewed in Refs. [13,14]. It has been considered in the context of bifurcation analysis in Ref. [12], which deals with torsion and the associated helical buckling bifurcation, and in Ref. [22] in the context of mixture theory for a particular case associated with bulging. The range of instability solutions for the particular problem at hand within its bifurcation spectrum is provided here.

The paper is organized as follows. In Section 2, the deformation produced in a circular cylindrical tube subjected to a uniform axial loading combined with a uniform internal pressure is described, along with the residually stressed elastic material model (constitutive law) of the structure. Both ends of the tube are open. Bifurcation from the deformed circular cylindrical geometry of the tube is analyzed. Three modes of bifurcation are possible: a prismatic mode in which the tube becomes noncircular with uniform cross-section; a bulging mode, which is axisymmetric; and a composite mode also known as bending bifurcation. In Section 3, a numerical methodology to analyze bifurcation and post-bifurcation of an inflated and extended residually stressed circular cylindrical tube subject to radial and circumferential residual stresses is provided. Numerical results are presented in Section 4. The dependence of bifurcation behavior of tubes under the loading at hand on residual stresses is illustrated and compared with results when there is no residual stress. Bending and bulging modes are captured. Bulging bifurcation is expected for sufficiently large values of the axial stretch. On the other hand for small values of the axial stretch, the onset of bifurcation is found to be the bending mode. Post-bifurcation is also studied. In the case of bending the structure in subsequent motion after the onset of bifurcation can support higher pressures until bulging is obtained. The bulge, or the abnormal enlargement, appears on one side of the tube showing an irregular shape which is consistent with the development of abdominal aortic aneurysm (AAA). Furthermore, it is shown that bending triggers bulging as opposed to the situation in which bending is not allowed and the onset of bifurcation is associated with bulging. This means that delayed aneurysm formation is possible if bending is not permitted. Nevertheless, in all cases for the material model at hand bulging instability propagates radially which would be associated with an aneurysm rupture because it cannot sustain more pressure. Some final remarks and conclusions are provided in Section 5.

\section{Mathematical description of the problem}

In this section, the mathematical description of the problem at hand is given. This includes geometry, material model, equilibrium equations and bifurcation of a thick-walled tube subjected to combined axial loading and internal pressure. Detailed equations of the finite deformation boundary-value problem for the residually-stressed elastic body at hand can be found in Ref. [13].

\subsection{Geometry}

We consider a circular tube of diameter $\mathrm{D}$, thickness $\mathrm{H}$, and length $\mathrm{L}$ which, in terms of cylindrical polar coordinates, $(R, \Theta, Z)$, is defined by

$A \leq R \leq B, \quad 0 \leq \Theta \leq 2 \pi, \quad-L / 2 \leq Z \leq L / 2$,

in the reference configuration $\boldsymbol{B}_{\mathrm{r}}$, where $A$ and $B$ are the internal and external radii. The position vector of a material point can then be written

$\mathbf{X}=R \mathbf{E}_{R}(\Theta)+Z \mathbf{E}_{Z}$

where $\mathbf{E}_{R}, \mathbf{E}_{\Theta}$ and $\mathbf{E}_{Z}$ are unit vectors in the indicated directions.

The cylinder is inflated and extended so that it remains a circular tube. The inflating pressure is denoted by $p$ and the axial load by $N$. In the deformed configuration $\mathbf{x} \in \mathcal{B}$ the cylinder is described by

$\mathbf{x}=r \mathbf{e}_{r}(\theta)+z \mathbf{e}_{z}, \quad a \leq r \leq b, \quad 0 \leq \theta \leq 2 \pi, \quad-\ell / 2 \leq z \leq \ell / 2$

where $(r, \theta, z)$, are cylindrical polar coordinates in this configuration. The deformed length of the cylinder is $\ell$. Referred to cylindrical coordinates, the associated deformation gradient tensor $\mathbf{F}$ for an incompressible material has components $\operatorname{diag}\left(\lambda_{r}, \lambda_{\theta}, \lambda_{z}\right)$, where $\lambda_{\theta}=r / R>0$ is the azimuthal principal stretch and $\lambda_{z}$ is the axial stretch, so that $\ell=\lambda_{z} L$. Because of the assumed incompressibility, the principal radial stretch is $\lambda_{r}=\lambda_{\theta}^{-1} \lambda_{z}^{-1}$. The displacement is $\mathbf{u}=\mathbf{x}-\mathbf{X}$.

\subsection{Equilibrium equations including residual stress}

Throughout this paper, we assume that there are no body forces present. The Cauchy stress $\sigma$ and the nominal stress $S$ then satisfy the equilibrium equations

$\operatorname{div} \boldsymbol{\sigma}=\mathbf{0}, \quad \operatorname{Div} \mathbf{S}=\mathbf{0}$,

respectively, where div and Div are the divergence operators with respect to $\mathrm{x} \in \mathcal{B}$ and $\mathrm{X} \in \mathcal{B}_{\mathrm{r}}$, respectively, and are connected by 
$\sigma=$ FS for the considered incompressible material. In the absence of intrinsic couple stresses, $\sigma$ is symmetric and hence $(\mathbf{F S})^{\mathrm{T}}=$ FS.

We now assume that the reference configuration $\mathcal{B}_{\mathrm{r}}$ is residually stressed, with the residual stress tensor denoted by $\sigma_{0}$. In this configuration $S=\sigma=\sigma_{0}$, i.e. there is no distinction between different measures of stress since the deformation is measured from $\mathcal{B}_{\mathrm{r}}$.

Residual stress arises in the absence of body forces and surface tractions on the boundary $\partial \mathcal{B}_{\mathrm{r}}$ of the material body $\boldsymbol{B}_{\mathrm{r}}$. It is also assumed that it is not accompanied by intrinsic couple stresses, so that it is symmetric $\left(\sigma_{0}^{\mathrm{T}}=\sigma_{0}\right)$ and therefore the rotational balance equations are satisfied in $\mathcal{B}_{\mathrm{r}}$ as well as the equilibrium equation

$\operatorname{div} \sigma_{0}=\mathbf{0}$.

Since there are no surface tractions, then, by definition, $\sigma_{0}$ must also satisfy the boundary condition

$\boldsymbol{\sigma}_{0} \mathbf{N}=\mathbf{0}$ on $\partial \mathcal{B}_{\mathrm{r}}$.

It follows that residual stresses are necessarily non-uniform and geometry dependent, therefore, the elastic response of a residually-stressed material body is inhomogeneous [21]. For the considered circular cylindrical geometry it is assumed that the only components of residual stress are $\sigma_{0 R R}, \sigma_{0 \Theta \Theta}$ and $\sigma_{0 Z Z}$, i.e. there is no residual shear stress, an assumption that is compatible with the boundary condition (5) that the residual stress must satisfy. However, the $Z$ component of the equilibrium equation and corresponding boundary conditions $\sigma_{0 Z Z}=0$ on the ends of the (finite length) tube then ensure that $\sigma_{0 z Z} \equiv 0$. The remaining components, $\sigma_{0 R R}$ and $\sigma_{0 \Theta \Theta}$, can then be taken to depend only on $R$, and the non-trivial component of the equilibrium Eq. (4) is the radial equation

$\frac{\mathrm{d} \sigma_{0 R R}}{\mathrm{~d} R}+\frac{1}{R}\left(\sigma_{0 R R}-\sigma_{0 \Theta \Theta}\right)=0$,

with accompanying boundary conditions from (5):

$\sigma_{0 R R}=0 \quad$ on $R=A, B$.

Note that if $\sigma_{0 R R}$ is known then $\sigma_{0 \Theta \Theta}$ is determined by (6) as $\mathrm{d}\left(R \sigma_{0 R R}\right) / \mathrm{d} R$.

\subsection{Material model}

The cylinder is made of an incompressible hyperelastic material with a constitutive equation given by a strain energy function $W$. For an isotropic material, the independent invariants are, in the case of an incompressible material,

$I_{1}=\operatorname{trC}, \quad I_{2}=\operatorname{tr}\left(\mathbf{C}^{-1}\right)$

where $\mathbf{C}=\mathbf{F}^{T} \mathbf{F}$ is the right Cauchy-Green deformation tensor, $\mathbf{F}$ is the deformation gradient, and tr denotes the trace of a second-order tensor. When the material is residually stressed, then it is also inhomogeneous. We consider its response still to be elastic relative to $\mathcal{B}_{\mathrm{r}}$, with dependence on $\mathbf{X}$ through $\sigma_{0}(\mathbf{X})$, and we account for this by including $\sigma_{0}$ in the argument of $W$. Thus, we write

$W=W\left(\mathbf{F}, \sigma_{0}\right)$

Note that this is automatically objective since $\sigma_{0}$ is unaffected by rotations in the deformed configuration $\boldsymbol{B}$ and $W$ depends on $\mathbf{F}$ only via $\mathbf{C}=\mathbf{F}^{\mathrm{T}} \mathbf{F}$. For an incompressible residually stressed material $W$ depends on nine invariants of $\mathbf{C}, \sigma_{0}$ and their combination. The particular strain energy function we consider here is

$W:=\frac{\mu}{2}\left(I_{1}-3\right)+\frac{f}{2}\left(I_{5}-\operatorname{tr} \sigma_{0}\right)+\frac{1-f}{4}\left(I_{6}-\operatorname{tr} \sigma_{0}\right)$,

where $I_{5}:=\operatorname{tr}\left(\sigma_{0} C\right), I_{6}:=\operatorname{tr}\left(\sigma_{0} C^{2}\right)$ and $\mu$ is a material constant. The value $f \in\{0,1\}$. If $f=0$, then (10) does not include the invariant $I_{5}$ while if $f=1$ it follows that (10) does not include the invariant $I_{6}$. If there is no residual stress $\left(\sigma_{0}=0\right)$ then the material model is just the well known neo-Hookean one. Here, the initial stresses that we impose in the strain energy function are (see Ref. [13])

$\sigma_{0 R R}=\bar{\beta}(R-A)(R-B)$ and

$\sigma_{0 \Theta \Theta}=\bar{\beta}\left[3 R^{2}-2(A+B) R+A B\right]$,

where $\bar{\beta}$ is the strength of the residual stress with units of stress per square length. It can be non-dimensional taking $\bar{\beta}=\mu \beta /(2 B T)$. This is an axisymmetric stress field with $\sigma_{0 z z}=0$.

There are no reliable data on residual stresses in AAA. Nevertheless, as pointed out in Ref. [22], these expressions are consistent residual stresses arising from the opening angle method. We will nondimensionalize our results so there is no need to specify values for the material constants. We refer to Ref. [23] for specific values of the residual stress.

We may now regard $W$ as a function of the above invariants. Thus, we take $W=W\left(I_{1}, I_{5}, I_{6}\right)$, and use the notation $W_{i}=\partial W / \partial I_{i}, i=1,5,6$ to write the Cauchy stress tensor as

$\sigma=2 W_{1} \mathbf{B}+2 W_{5} \boldsymbol{\Sigma}+2 W_{6}(\boldsymbol{\Sigma} \mathbf{B}+\mathbf{B} \boldsymbol{\Sigma})-q \mathbf{I}$,

in which we have introduced the notation $\boldsymbol{\Sigma}=\mathbf{F} \sigma_{0} \mathbf{F}^{\mathrm{T}}$ for the Eulerian tensor which is the push forward of $\sigma_{0}$ from $\boldsymbol{B}_{\mathrm{r}}$ to $\mathcal{B}$ and q is the Lagrange multiplier due to the incompressibility constraint. We also recall that $\mathbf{B}=\mathbf{F F}^{\mathrm{T}}$ is the left Cauchy-Green tensor.

\subsection{Bifurcation of thick-walled tubes}

The equilibrium equations for models with no residual stress can be obtained, for instance, by minimizing the energy functional of this purely mechanical analysis. Results show that curves giving the applied pressure against the azimuthal stretch (in a more general sense loaddisplacement curves) reach instability points which are close to maximum points of these curves. In the case of thick-walled tubes, this analysis has been given in Ref. [3].

To investigate the possible bifurcation modes of the tube one should consider incremental displacements with respect to the deformed configuration (under equilibrium), of the general form

$\delta \mathbf{u}=\delta u_{r}(r, \theta, z) \mathbf{e}_{r}+\delta u_{\theta}(r, \theta, z) \mathbf{e}_{\theta}+\delta u_{z}(r, \theta, z) e_{z}$.

There are essentially three distinct possible bifurcation modes. A prismatic mode where the incremental displacement is assumed to be independent of z. One may take $\delta u_{z}$ to be zero without any loss of generality but the length dimension of the cylinder is lost. Secondly, a bulging mode where the incremental displacements are assumed to be independent of $\theta$ and one may take $\delta u_{\theta}$ to be zero. Thirdly the more general case, the composite mode, where no assumptions of the form of $\delta \mathbf{u}$ are made. In general, we just note that the solution procedure requires the solution of different homogeneous (fourth or higher) order ordinary differential equations with four second-order boundary conditions. This analysis involves a complicated mathematical machinery. In the next section, we develop a methodology that can be used easily in engineering analysis and design. The finite element method is a helpful tool to handle and tackle this problem. In what follows we provide more information about the bulging and bending modes, which are the ones captured in our numerical analysis.

\subsubsection{Bulging mode}

Bulging mode is a bifurcation mode of an inflated tube in which it remains a circular one. To obtain the mathematical formulation, an axisymmetric displacement field $\left(\delta u_{\theta}=0\right)$ of the form

$\delta \mathbf{u}=\delta u_{r}(r, z) \mathbf{e}_{r}+\delta u_{z}(r, z) \mathbf{e}_{z}$

needs to be considered.

Among the instability modes of stretched pressurized cylinders bulging is likely to be the first that appears. Our effort here is to analyze 
the dependence of bifurcation on the behavior of residually stressed tubes under the loading at hand. Results show qualitative changes for the onset of bifurcation when residual stresses are considered as opposed to the situation in which residual stresses are not considered. Post-bifurcation, i.e. bulging motion, also attracts our attention. It has been shown that bulging instability propagates either axially or radially and that it depends on the material model. For axial propagation of bulging pressure of inflation must be increased and the structure in this subsequent motion can support higher pressures than the one associated with bulging nucleation. This is in agreement with the possible propagation of aneurysms in arterial wall tissue. On the other hand, radial expansion of bulging is related to a decrease of pressure beyond the onset of bulging and it is related to aneurysm rupture. Propagation of the instability mode for the residually stressed materials will also be analyzed. For further details on post-bifurcation we refer to Ref. [6].

\subsubsection{Bending mode: combination of prismatic mode and bulging mode}

Blood vessels, such as retinal arteries and veins, often become tortuous along their paths. This could be associated with hypertension, diabetes, and retinopathy (see Refs. $[18,19]$ ). It has been shown that reducing axial extension in arteries can lead to the development of tortuous shape [20]. These are factors that modify the artery as a perfect circular cylindrical geometry. In addition, there are arteries that undergo significant bending, such as the superficial femoral artery. Specialized stent designs are needed in these arteries due to the large bending deformations [28]. When the artery shows significant bending saccular aneurysms seem to appear [28]. But even AAA shows that the bulge (abnormal enlargement) develops in one side of the tube and this is not captured just with the axisymmetric bulging instability analysis. It is important to study different factors associated with the bending of an artery. In our analysis, great insight will be given in the next section with the bending instability and it is important to describe here its mathematical formulation.

We consider the bending instability of a perfect circular cylindrical membrane under internal pressure and axial loading. The combination of prismatic and bulging mode may be interpreted in terms of bending. Fig. 1 shows the post-bifurcation shape of the bending instability.

We summarize the equations for a cylindrical membrane (i.e. $\sigma_{r r}=0$ and no residual stress) since in the next sections we plot together, accordingly, results for a membrane with our numerical results for a thick-walled tube to simultaneously guide and validate our numerical results. For further details, we refer to Ref. [2]. For this particular problem, one can write

$\sigma_{\theta \theta}=\lambda_{\theta} \frac{\partial \widehat{W}}{\partial \lambda_{\theta}} \quad$ and $\quad \sigma_{z z}=\lambda_{z} \frac{\partial \widehat{W}}{\partial \lambda_{z}}$

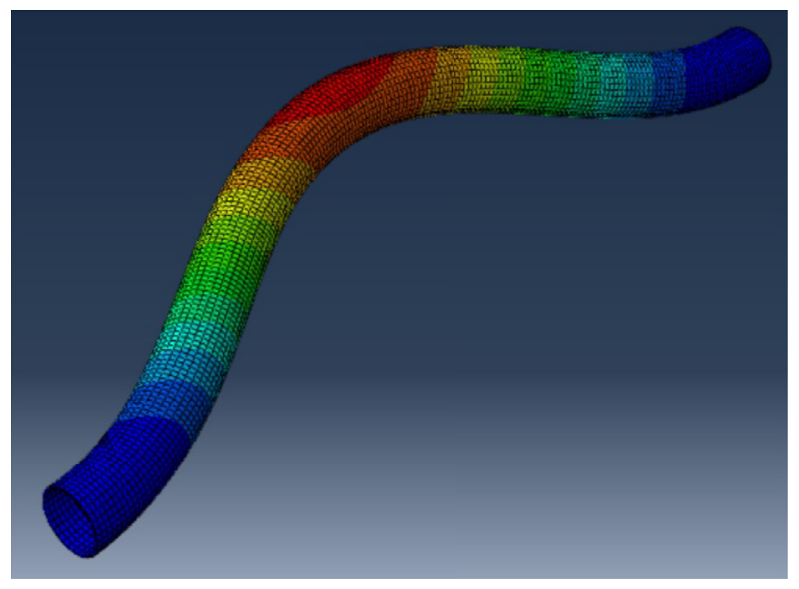

Fig. 1. Post-bifurcation shape of the bending mode for $\lambda_{z}=1, \beta=0.5$, and $f=0$. where we have introduced the notation $\widehat{W}\left(\lambda_{\theta}, \lambda_{z}\right)=$ $W\left(\lambda_{\theta}^{-1} \lambda_{z}^{-1}, \lambda_{\theta}, \lambda_{z}\right)$. The equilibrium equations for a membrane give the inflating pressure in the form

$p=\frac{H \widehat{W}_{\lambda_{\theta}}}{R \lambda_{\theta} \lambda_{z}}$,

where $\widehat{W}_{\lambda_{\theta}}$ denotes $\frac{\partial \widehat{W}}{\partial \lambda_{\theta}}$. The onset of bending instability can be written

$\sigma_{\theta \theta}-2 \sigma_{z z}=0$

It follows that unstable configurations obey $\sigma_{\theta \theta}-2 \sigma_{z z}>0$ as given in Ref. [2]. This criterion is used for thick-walled tubes to guide the numerical analysis. There is no analytical solution for bending bifurcation under those circumstances.

\section{Finite element analysis}

Finite element analyses are developed from the so-called weak formulations of the problem, i.e. energy functionals. The problem at hand is transformed into a system of algebraic equations in which the displacements are involved. This is a well-established procedure and we will not develop the formulation. Here we describe the numerical technique developed, and, for the purpose of this communication, we focus on the commercial code Abaqus [25]. We use a software package for professional purposes since one of the main objectives of this work is to disseminate and promote nonlinear computational modeling techniques.

\subsection{Model data: geometry and material model}

We have modeled the constitutive law introduced previously and our goal is to analyze the combined extension and inflation of a circular cylindrical tube subject to radial and circumferential residual stresses. The computational methodology developed in Ref. [4] paid attention to the onset of bifurcation with no residual stress. Residual stress was the subject of [12] in the context of torsion and helical buckling.

We consider a cylinder with $L / D=20$ and $H / D=0.05$. In particular, the model is a cylinder with the length of $200 \mathrm{~mm}$, diameter of $10 \mathrm{~mm}$ and thickness of $0.5 \mathrm{~mm}$. The geometry has been meshed with three-dimensional hybrid linear solid elements (C3D8RH in the Abaqus notation). In general, the solution to a problem that deals with incompressible material behavior cannot be obtained in terms of the displacement history only, since a purely hydrostatic pressure can be added without changing the displacements. The hybrid formulation available in Abaqus solves this problem removing this behavior of the system. More in particular, the hybrid formulation treats the pressure as an independent interpolated basic solution variable which is coupled, using a Lagrange multiplier, to the displacement solution through the constitutive theory and the compatibility condition. In addition, this formulation avoids the problem of volume (strain) locking, which occurs when the finite element mesh cannot properly represent incompressible deformations. Five elements are taken in the thickness of the tube to capture the residual stress field. In the azimuthal direction, two situations are distinguished: for bending instability, 80 elements are taken while 32 elements are considered when bulging instability is expected. In the axial direction, different numbers of elements varying from 200 to 800 are considered for the different simulations according to the different axial stretches. A detailed view of the mesh is shown in Fig. 2.

The material model has been implemented in Abaqus as a subroutine (UMAT) to be used for incompressible hybrid elements. The user must provide the Cauchy stress tensor $\sigma$. In particular, the deviatoric stress must be provided for a fully incompressible user material (see Ref. [25]). The incompressibility condition is then imposed by the finite element code using a Lagrange multiplier. For further details, we refer to Ref. [12]. 


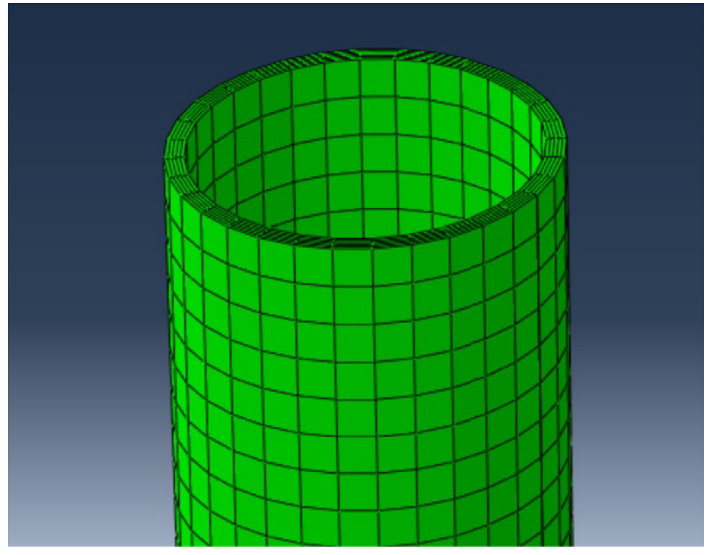

Fig. 2. Detailed finite element mesh of the tube.

\subsection{Numerical procedure}

The numerical procedure developed is just summarized. Each simulation requires three basic steps. Residual stresses are generated during a first step. Numerically some iterations might be needed to establish the initial equilibrium of the residually stressed tube. To enhance convergence decreasing the time step the strength of the residual stress $\beta$ is linearly increased from zero to its actual value. In our case, only one increment with two iterations were necessary to establish the equilibrium. The von Mises residual stress at the end of the first step is plotted in Fig. 3. The radial and hoop residual stresses along the thickness direction are shown in Fig. 4. Second, we stretch the cylinder by imposing relative axial displacements. Hoop displacements along the tube are prevented in a nodal local cylindrical system during the first and second step. A circular cylindrical tube is obtained. In the third step, we apply internal pressure in a modified Riks analysis (arc length procedure [24]). In this step, the load is the inner pressure with a magnitude of $p=10 * \mu * H / D$. We distinguish two situations with respect to displacement restrictions. To capture just bulging hoop displacements should be prevented as it has been done during the first and second step. On the other hand, to capture other instability modes just hoop and axial displacements should be restricted at the ends of the tube. Any other movement is allowed along the tube.

The modified Riks method introduces two concepts: the load proportional factor (LPF) and the arc length, for details see Ref. [24]. The arc length is a quantity related to the evolution of the structure that

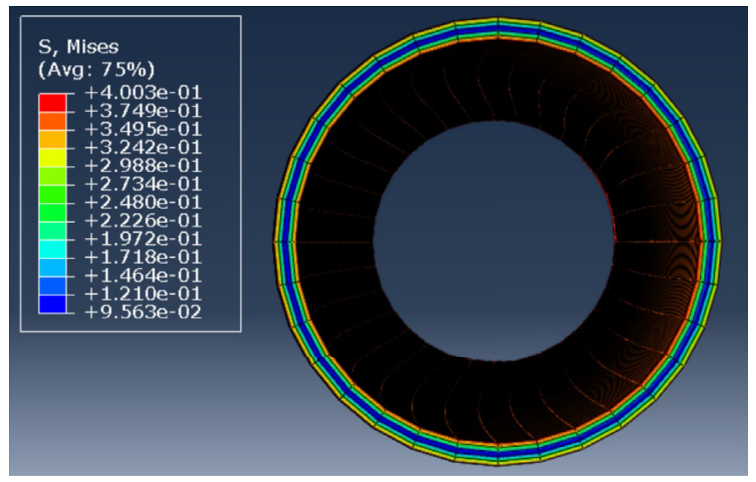

Fig. 3. Contour plots of the von Mises stress are presented to show the formation of the residual stress distribution at the last increment of the first step. Values are normalized with respect to the material constant $\frac{\mu}{2}$.

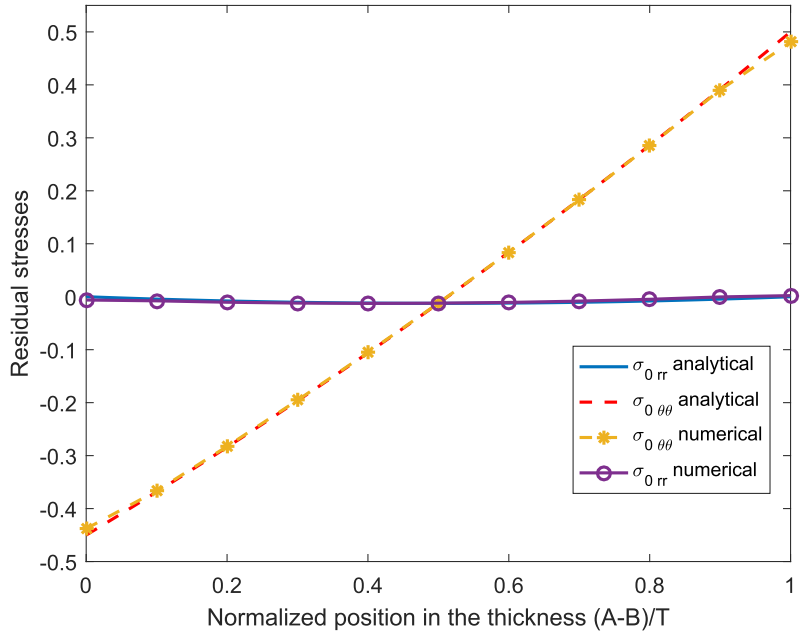

Fig. 4. Normalized radial and hoop residual stresses (normalized with respect to the material constant $\frac{\mu}{2}$ ) along the normalized thickness of the tube. The numerical values are the ones given by the numerical computations using abaqus. The analytical ones are given by Eqs. (11) and (12). The curves show that corresponding numerical and analytical values coincide.

combines displacements and loads. The load proportional factor determines the load applied and it may decrease. In fact, Riks method is not very useful when the load proportional factor is monotonically increasing. Unlike the Newton-Method, the Arc Length (modified Riks) method postulates a simultaneous variation in both the displacements and the load vector coefficient.

In Fig. 5 a typical curve of the load proportional factor vs the arc length is shown. In this curve, there is a maximum which is an indicator that the system exhibits an instability. The way to understand this curve is the following. LPF is related to the load applied. As loading is applied the structure will reach the maximum point shown in the curve in a sequence of states under static equilibrium. It is not possible to increase the load above the maximum LPF under static equilibrium. Furthermore, a sudden and new catastrophic rearrangement could be obtained by the structure for loads close to the maximum LPF. This is an indicator of an instability but it should not be understood as a bifurcation point. To capture bifurcation, an imperfection has to be introduced in the geometry of the problem. That way, the structure will behave smoothly as loading approaches the instability point.

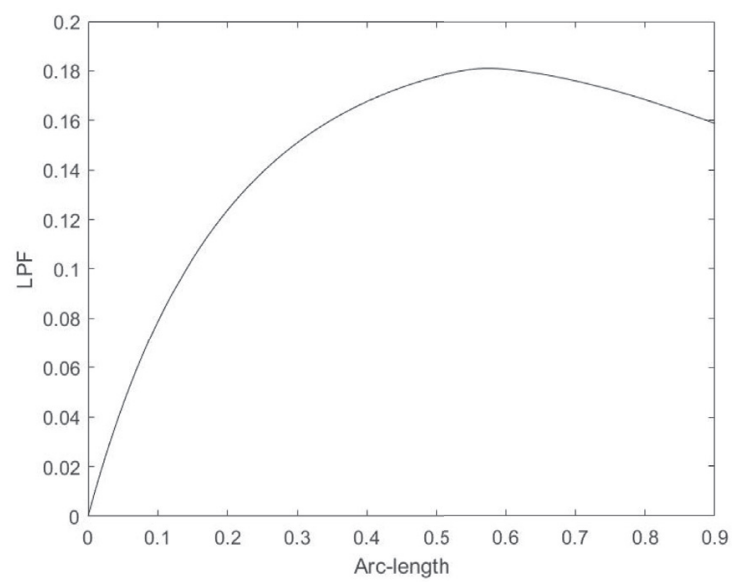

Fig. 5. Values of the LPF vs. arc length showing a maximum, which is associated with an instability. 
The onset of bulging bifurcation is determined in the following way. To capture bulging, one can follow the evolution (with deformation) of the hoop stretch of two points which are located in the middle of the thickness of the cylinder at two different cross sections (at two different altitudes). One point is outside the bulge and we refer to it as out, and the other point is inside the bulge, and we refer to it as in. As loading is applied, bulging bifurcation is identified when the hoop stretches of these two points differ. In particular, we take the bifurcation point when the deviation of the hoop stretches of these two points is a small fraction of the hoop stretch average of both points (for instance, $1 \%$ ). This procedure also allows us to follow post-bifurcation. To capture bending, in general, two conditions help to capture the onset of the bending mode. First, in analogy with the membrane analysis in which unstable configurations obey $\sigma_{\theta \theta}-2 \sigma_{z z}>0$ we study that particular condition along the length of the tube as loading is applied. In our case, with a thick-walled tube, the values of stresses considered in that condition are average stresses at the cross sections of the tube. Second, the (radial) displacement analysis of the cylinder also helps to establish the onset of bending, which is associated with a particular mode of this instability. Our numerical results in the next section show that the onset of bending is associated with the mode $m=1$ case (see Ref. [3] for details). Fig. 1 shows this mode of bifurcation.

\section{Numerical results}

The values of stress (the components of $\sigma$ ) presented in this section are normalized with respect to the material constant $\ddot{2}_{2}$ and we avoid repetition indicating this now. To avoid a strict numerical bifurcation, we introduced a small imperfection at the center of the tube with a maximum deviation of $0.001 B$ with respect to the perfect circular cylinder (see for further details [5]). We consider 5 different cases in terms of residual stresses: one with no residual stress $(\beta=0)$, and four with residual stresses corresponding to the combination of the values of $f \in\{0,1\}$ and $\beta \in\{-0.5,0.5\}$. A positive (negative) value of $\beta$ is associated with tensile (compressive) residual hoop stress in the outer wall of the tube.

Each simulation has a particular value of $\beta, f$ and $\lambda_{z}$. Our numerical results show that the onset of bifurcation is either bulging or bending. Furthermore, the onset of bending is associated with the mode $\mathrm{m}=1$. The following description distinguishes the two bifurcation scenarios. Consider the simulations (i) $\lambda_{z}=1, \beta=0.5$ and $f=0$ and (ii) $\lambda_{z}=1.4, \beta=0.5$, and $f=0$. To get a first approximation to the bifurcation scenario in each case values of $\sigma_{\theta \theta}-2 \sigma_{z z}$ vs arc length are plotted in Fig. 6 . The values $\sigma_{\theta \theta}-2 \sigma_{z z}$ are average stresses of the tube middle cross-section. For case (ii), i.e. $\lambda_{z}=1.4$, Fig. 6 shows that $\sigma_{\theta \theta}-2 \sigma_{z z}<0$ (dashed line), which can be taken as a sign that bending is not expected to give the onset of bifurcation. In fact, our numerical solution gives bulging. On the other hand, in Fig. 6 there are values obeying $\sigma_{\theta \theta}-2 \sigma_{z z}=0$ for case (i), solid line. This indicates that bending may give the onset of bifurcation although this is not enough to establish the bifurcation scenario. Attention is paid to the following. Values of LPF vs arc length for case (i) are shown in Fig. 7, dashed line. As it is explained in the previous section one can restrict the analysis to capture just bulging and we consider case (i) under those circumstances. The corresponding values of LPF vs arc length for this simulation are also shown in Fig. 7, solid line. A simple observation of the two curves given in Fig. 7 shows that as loading is applied when the dashed line (bending) reaches its maximum the solid line (bulging) still increases monotonically. This indicates that bending is associated with initial bifurcation for case (i) and therefore that the onset of bifurcation is not bulging. Furthermore, bending bifurcation occurs when the two lines of the Figure separate. The maximum point of the dashed line is associated with the formation of a bulge (after bending), which will be explained later in detail.

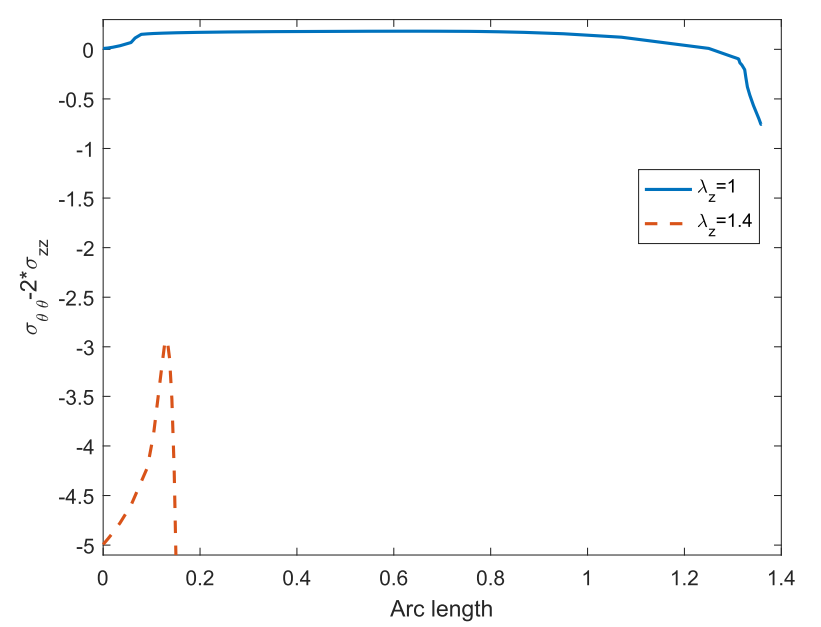

Fig. 6. Values of $\sigma_{\theta \theta}-2 \sigma_{z z}$ (average stresses of the tube middle cross section) vs arc length for the models $\lambda_{z}=1, \beta=0.5, f=0$, solid line, and $\lambda_{z}=1.4$, $\beta=0.5, f=0$, dashed line. For the former case, the curve shows values obeying $\sigma_{\theta \theta}-2 \sigma_{z z}>0$, which indicates that the onset of bifurcation may be associated with bending. On the other hand, for the latter case the curve shows that $\sigma_{\theta \theta}-2 \sigma_{z z}<0$, which indicates that bending is not a bifurcation mode under these conditions.

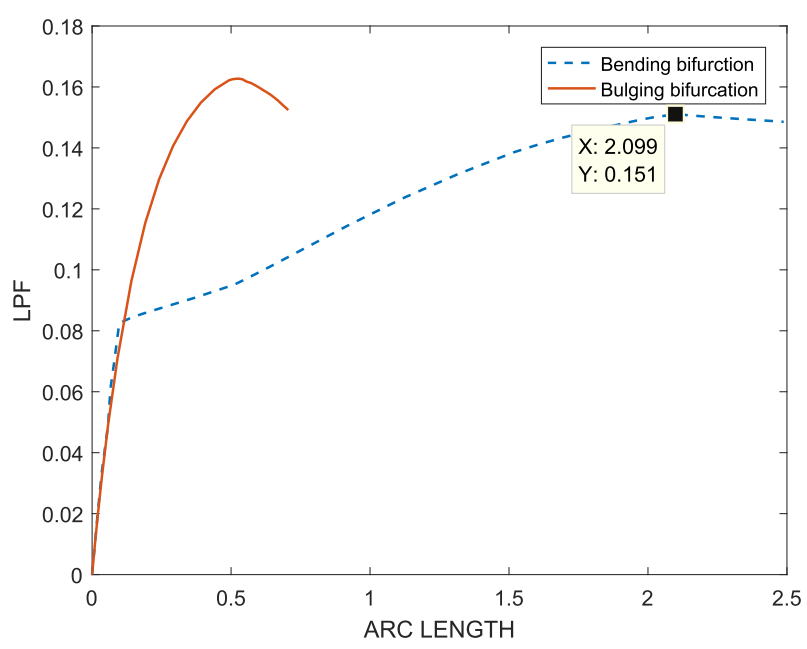

Fig. 7. Values of LPF vs arc length for the model $\lambda_{z}=1, \beta=0.5$ and $f=0$ when a) the simulation is restricted to capture just bulging, solid line, and b) the simulation is not restricted, dashed line. As loading is applied the dashed line (bending) reaches its maximum while the solid line (bulging) still increases monotonically. This indicates that bulging is not the onset of bifurcation. Furthermore, bending bifurcation occurs when the two lines separate. The maximum point of the dashed line is associated with the formation of a bulge (after bending).

To further illustrate the numerical solutions for (i) $\lambda_{z}=1$, $\beta=0.5$, and $f=0$ and (ii) $\lambda_{z}=1.4, \beta=0.5$, and $f=0$ we focus now on the stresses prior to the onset of bifurcation in each case. Fig. 8 shows values of azimuthal and axial average stresses on each crosssection along the tube length for the case (i) prior to the bifurcation, which is bending. Fig. 9 shows values of azimuthal and axial average stresses on each cross-section along the tube length for the case (ii) prior to the bifurcation, which is bulging. These two Figures are representative of other simulations for other values of $\beta$ and $f$.

Let us generalize the initial findings of this study. To investigate further the effect of residual stress, let us consider first for illustrative pur- 


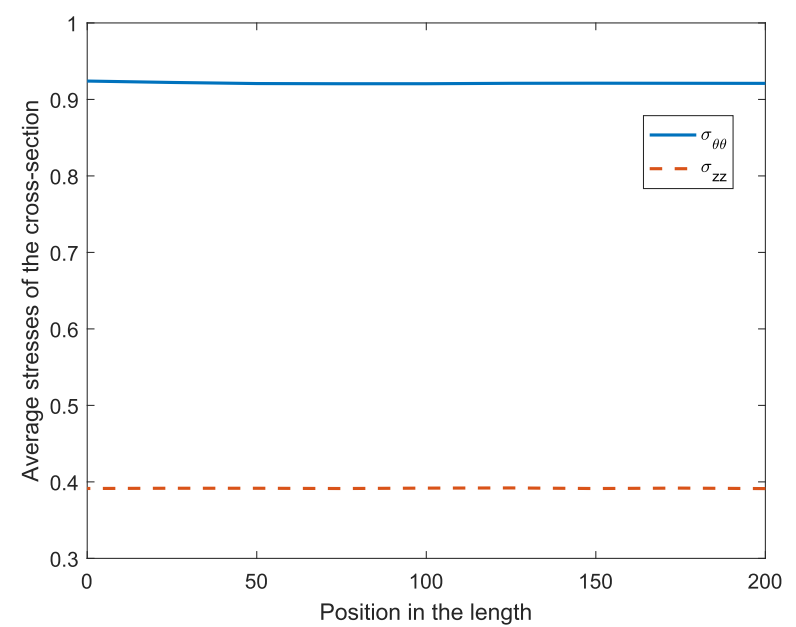

Fig. 8. Values of azimuthal and axial average stresses on each cross-section along the tube length for $\beta=0.5, f=0$ and $\lambda_{z}=1$ prior to the bifurcation, which is bending.

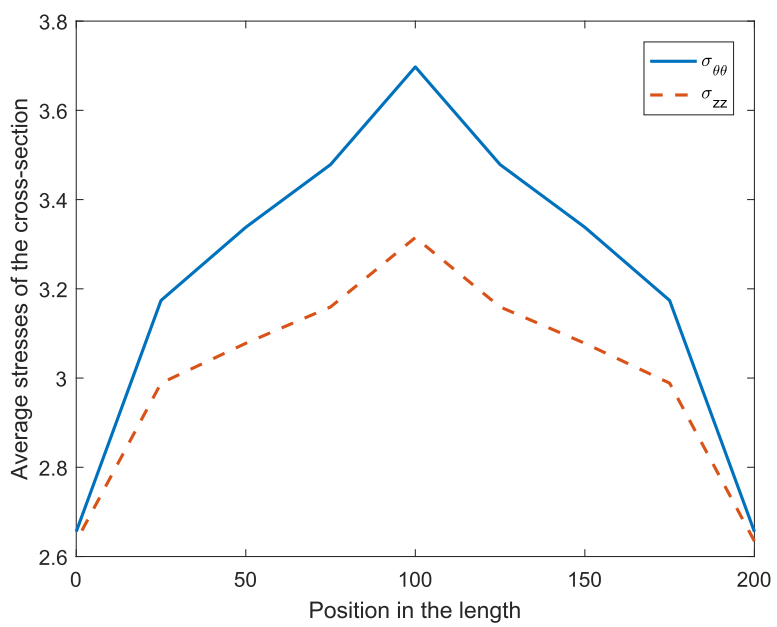

Fig. 9. Values of azimuthal and axial average stresses on each cross-section along the tube length for $\beta=0.5, f=0$ and $\lambda_{z}=1.4$ prior to the bifurcation, which is bulging.

poses simulations that only capture bulging (as it has been explained previously) for the five models at hand. Results of these analyses are given in Fig. 10, which gives values of pressure vs axial stretch at the onset of (bulging) bifurcation. On the other hand, corresponding results for simulations that are not restricted to capture just bulging are shown in Fig. 11. In both Figures, values for the case with no residual stress, $\beta=0$, are the same, as it is expected. On the other hand, the difference between Figs. 11 and 10 for the other cases reveal the effect of residual stress on the initiation of bifurcation. For all these four cases, a comparison between the curves in Fig. 11 and their corresponding ones in Fig. 10 indicates that the onset of bifurcation for sufficiently small values of $\lambda_{z}$ is not bulging. It can be observed in Fig. 11 that this domain of values obeys approximately $1.2>\lambda_{z} \geq 1$. Numerical calculations show that the onset of bifurcation for this domain is bending and, furthermore, that this bending mode is $m=1$. For values outside of that particular domain in $\lambda_{z}$, both figures are identical, which indicates that bifurcation is associated with bulging. There are no quantitative differences at the onset of bending for the models with residual stress as it

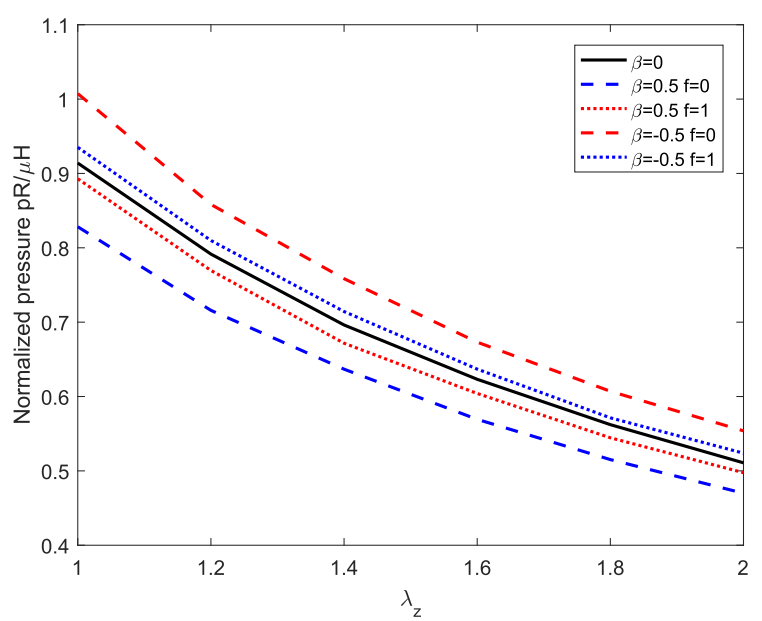

Fig. 10. Values of normalized inflation pressure vs axial stretch at the onset of bulging mode for the five material models at hand: one with no residual stress ( $\beta=0$ ), and four with residual stresses corresponding to the combination of the values of $f \in\{0,1\}$ and $\beta \in\{-0.5,0.5\}$. For a particular value of $\lambda_{z}$, there are quantitative differences among the four cases that include residual stresses. It follows that the values of pressure at bulging bifurcation for the models with tensile residual hoop stress in the outer wall of the tube (positive value of $\beta$ ) are lower than the values of pressure at bulging initiation for the models with compressive residual hoop stress in the outer wall of the tube (negative value of $\beta$ ). A similar effect that depends on the sign of $\beta$ occurs when the models $f=0$ and $f=1$ are compared. If $\beta$ is positive the values of pressure at bifurcation for the model with the invariant $I_{6}(f=0)$ are lower than the values of pressure at bifurcation for the model with the invariant $I_{5}(f=0)$. Conversely, if $\beta$ is negative the values of pressure at bifurcation for the model with the invariant $I_{6}(f=0)$ are higher than the values of pressure at bulging for the model with the invariant $I_{5}(f=0)$.

is shown in Fig. 11 where the curves of all these four cases are very close to each other. On the other hand, for a particular value of $\lambda_{z}$, there are quantitative differences among these four cases when bifurcation is associated with bulging. It follows that the values of pressure at bulging bifurcation for the models with tensile residual hoop stress in the outer wall of the tube (positive value of $\beta$ ) are lower than the values of pressure at bulging initiation for the models with compressive residual hoop stress in the outer wall of the tube (negative value of $\beta$ ). A similar effect that depends on the sign of $\beta$ occurs when the models $f=0$ and $f=1$ are compared. If $\beta$ is positive the values of pressure at bulging bifurcation for the model with the invariant $I_{6}(f=0)$ are lower than the values of pressure at bulging bifurcation for the model with the invariant $I_{5}(f=0)$. Conversely, if $\beta$ is negative the values of pressure at bulging bifurcation for the model with the invariant $I_{6}$ $(f=0)$ are higher than the values of pressure at bulging bifurcation for the model with the invariant $I_{5}(f=0)$.

More insight can be gained into the application of this work to aneurysm formation and propagation by analyzing post-bifurcation. First, let us consider post-bifurcation of bulging. In all cases for the material models at hand, once the bulge appears, it propagates radially which would be associated with an aneurysm rupture since it cannot sustain more pressure. This is illustrated in Fig. 12 in which values of pressure vs hoop stretch when $\lambda_{z}=1.2$ for different material models are shown. The membrane analytical solution given by (17) prior to bifurcation is shown (solid line) for both comparison and guidance of the results. More in particular, the Figure shows for each case the evolution (with deformation) of the hoop stretch of two points which are located in the middle of the thickness of the cylinder at two different cross sections (at two different altitudes). One point is outside the bulge and we refer to it as out, and the other point is inside the bulge, and we 


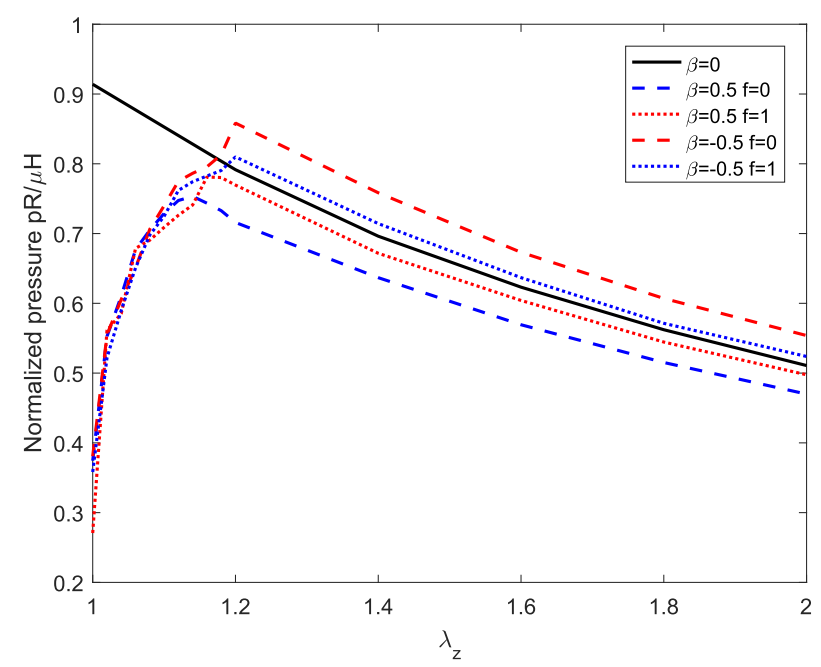

Fig. 11. Values of normalized inflation pressure vs axial stretch at bifurcation for the different models shown in Fig. 10, which gives only values associated with bulging. Figs. 10 and 11 show the same values for the case with no residual stress, $\beta=0$. This means that under these conditions the onset of bifurcation is bulging. With respect to the other four cases, a comparison between corresponding curves (same material model) in Figs. 11 and 10 indicates that all these four curves are different for values that obey approximately $\lambda_{z}<1.2$, while for $\lambda_{z}>1.2$ corresponding curves are identical. The latter assertion means that under those circumstances, the onset of bifurcation is bulging while the former one means that the onset of bifurcation is not bulging. Numerical calculations show that the onset of bifurcation is bending and, furthermore, that the bending mode is $m=1$.

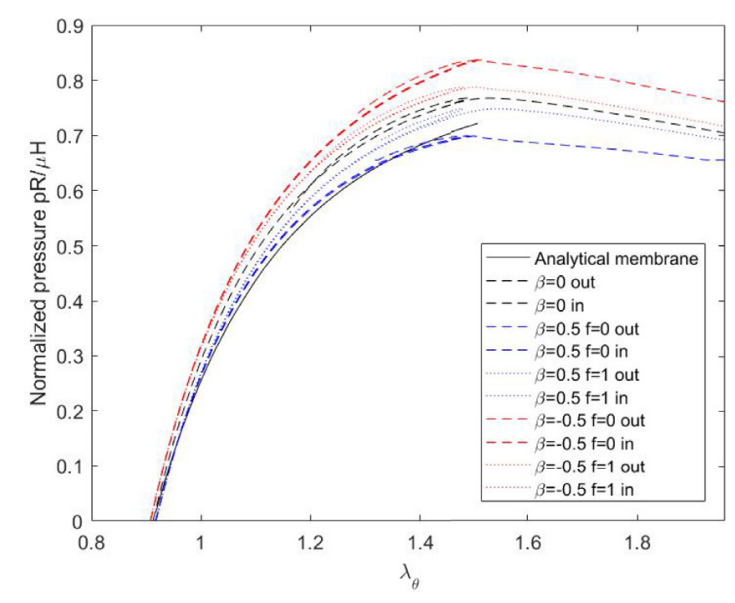

Fig. 12. For $\lambda_{z}=1.2$ and a particular model, i.e. fixing $\beta$ and $f$, the plot gives values of normalized pressure of inflation vs values of hoop stretch of two points which are located in the middle of the thickness of the cylinder at two different cross sections (at two different altitudes). As loading is applied, bulging bifurcation is identified when the hoop stretches of these two points differ. One point is outside the bulge and we refer to it as "out", and the other one is inside the bulge, and we refer to it as "in". When bifurcation occurs (close to maximum points in each curve), the structure is not able to support higher values of pressure. Furthermore, as pressure decreases, the point "out" of the bulge reverses the original path decreasing its hoop stretch while the one "in" the bulge increases its hoop stretch. When bifurcation occurs (close to maximum points in each curve), the structure is not able to support higher values of pressure. Furthermore, as the pressure decreases, the point out of the bulge reverses the original path decreasing its hoop stretch while the one in the bulge increases its hoop stretch. The membrane analytical solution is given by (17) prior to bifurcation is also shown (solid line).

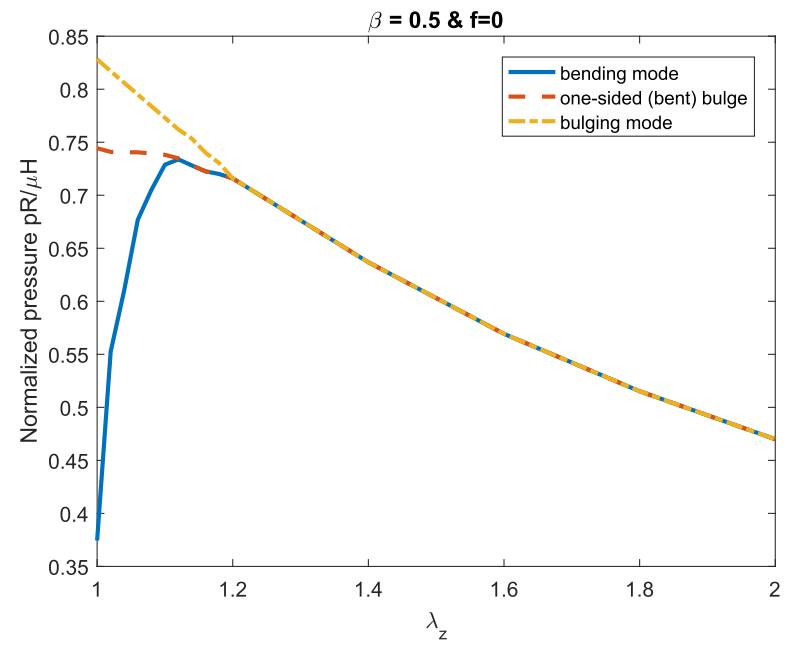

Fig. 13. Normalized inflation pressure at bifurcation against axial stretch for the case $\beta=0.5$ and $f=0$. In this Figure for sufficiently small values of $\lambda_{z}$ there are three curves. The solid line (bending) is the one given in Fig. 10. The dot-dashed line (bending is not allowed) is the one given in Fig. 11. The other line (dashed) in the Figure gives the formation of a bulge, which is triggered by the initial bending bifurcation. This bulge, or the abnormal enlargement, appears on one side of the tube showing an irregular shape which is consistent with the development of abdominal aortic aneurysm (AAA).

refer to it as in. As loading is applied, bulging bifurcation is identified when the hoop stretches of these two points differ. Prior to bifurcation, both points have a non-distinguishing hoop stretch difference. Under these conditions, the Figure shows in each numerical simulation (each cylinder) two lines that are very close. When bifurcation occurs (close to maximum points in each curve), the structure is not able to support higher values of pressure. Furthermore, as pressure decreases, point out of the bulge reverses the original path decreasing its hoop stretch while the point in the bulge increases its hoop stretch. The bulge is not able to support higher pressures than the one associated with the onset of bulging. It follows that the bulge propagates radially, which would be associated with an aneurysm rupturing.

Let us focus now on post-bifurcation for bending. Initiation of bending bifurcation is always associated with the mode $m=1$. The structure in subsequent motion can support higher pressures until bulging is obtained. The bulge, or the abnormal enlargement, appears on one side of the tube showing an irregular shape which is consistent with the development of abdominal aortic aneurysm (AAA). Furthermore, it is shown that bending triggers bulging, i.e. the values of pressure associated with the formation of a bulge are lower than the values of pressure associated with the simulation in which bending is not allowed and the onset of bifurcation is bulging. This is illustrated in Fig. 13 for the case $\beta=0.5$ and $f=0$. In this Figure for sufficiently small values of $\lambda_{z}$ there are three curves. The solid line (bending) is the one given in Fig. 10. The dot-dashed line (bending is not allowed) is the one given in Fig. 11. The other line (dashed) in the Figure gives the formation of a bulge, which is triggered by the initial bending bifurcation. Under these circumstances, the bulge, or the abnormal enlargement, appears on one side of the tube showing an irregular shape which is consistent with the development of abdominal aortic aneurysm (AAA). It follows that for sufficiently small values of $\lambda_{z}$ delayed aneurysm formation is possible if bending is not permitted. Nevertheless, for the material models at hand the bulge propagates radially.

For completeness, several representative histories of configurations showing bifurcation onset as well as bifurcation motion are given in the following Figures. In particular, Fig. 14 shows a graphic representation of the bulging onset and motion. The material model of this Figure is just the well known neo-Hookean one, i.e. with no residual stress. 


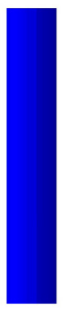

(1)

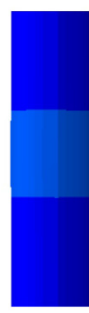

(2)

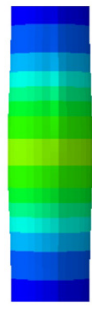

(3)

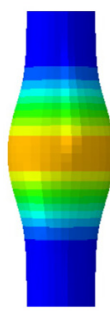

(4)

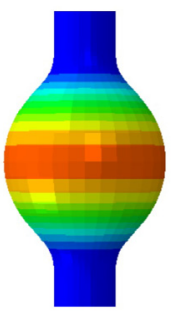

(5)

Fig. 14. History of configurations, (1) is the beginning and (5) is the ending, associated with bulging bifurcation for the material $\beta=0$ and $f=0$ with $\lambda_{z}=1$. This case is a graphic representation of the bulging motion.

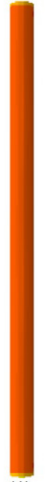

(1)

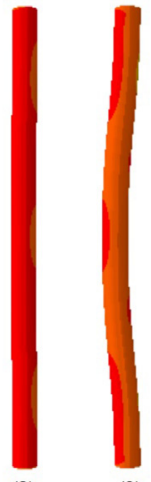

(3)

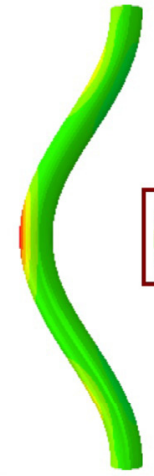

(4)

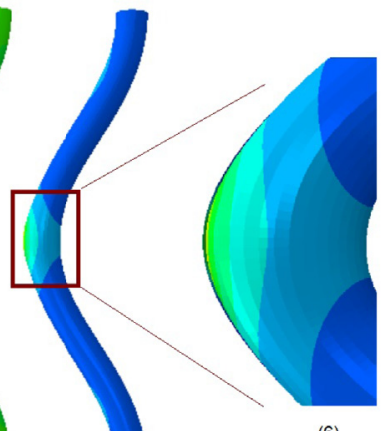

(6)

Fig. 15. History of configurations associated with bending for $\beta=0.5, f=0$ and $\lambda_{z}=1$. (1) is the beginning of inflation and (5) is the ending of it. (6) is a magnification of the one-sided bulge, which propagates radially.

Nevertheless, as it has been explained previously, bifurcation onset and motion for models with residual stresses under sufficiently large values of $\lambda_{z}$ follow the same qualitative description. Now, let us consider residual stresses. We take the material model illustrated in Fig. 13, i.e. $\beta=0.5$ and $f=0$, and some (representative) values of $\lambda_{z}$ increasing from $\lambda_{z}=1$ (Fig. 15) to $\lambda_{z}=1.18$ (Fig. 18), which is close to the value $\lambda_{z}=1.2$, associated with bulging. As it is shown in Fig. 13, in all cases, bending gives the onset of bifurcation, with bending mode $m=1$, and in subsequent motion a one-sided bulge that propagates radially forms. Figs. 15-18 reveal the transition from the onset of bend-

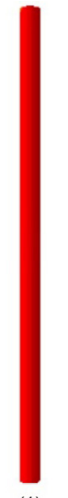

(1)

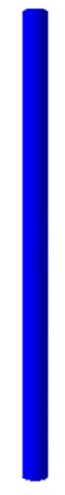

(2)

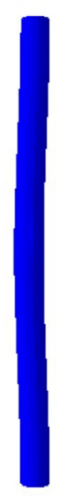

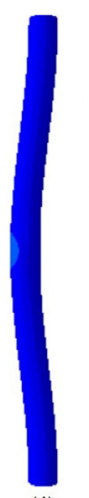

(4)

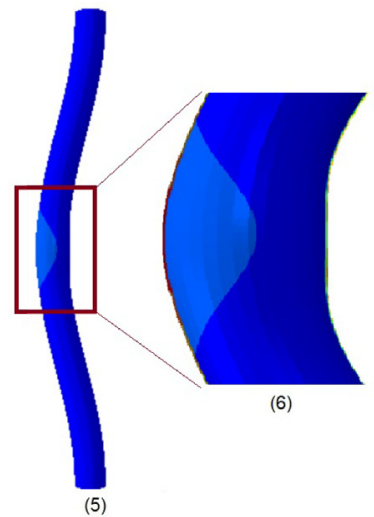

Fig. 16. History of configurations associated with bending for $\beta=0.5, f=0$ and $\lambda_{z}=1.04$. (1) is the beginning of inflation and (5) is the ending of it. (6) is a magnification of the one-sided bulge, which is propagating radially.
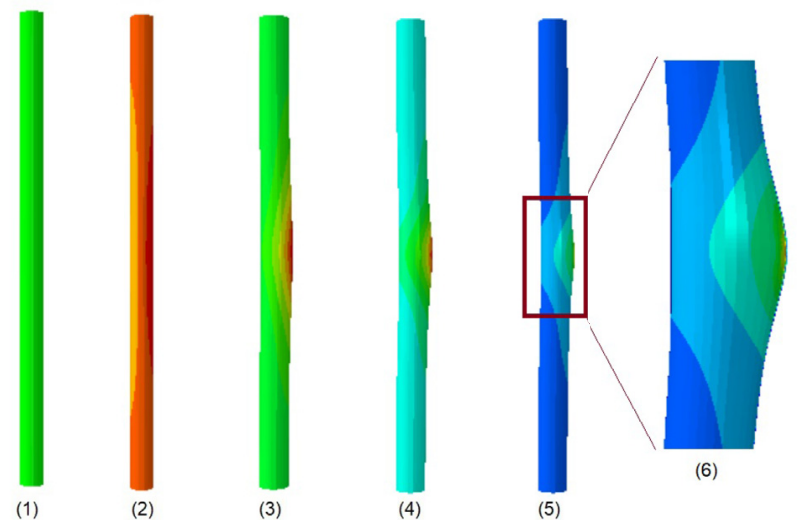

Fig. 17. History of configurations associated with bending for $\beta=0.5, f=0$ and $\lambda_{z}=1.12$. (1) is the beginning of inflation and (5) is the ending of it. (6) is a magnification of the one-sided bulge, which is propagating radially.
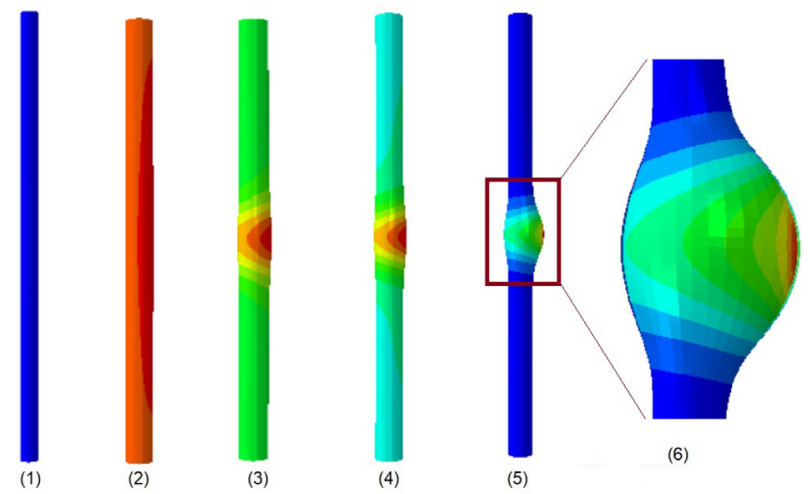

Fig. 18. History of configurations associated with bending for $\beta=0.5, f=0$ and $\lambda_{z}=1.18$. (1) is the beginning of inflation and (5) is the ending of it. (6) is a magnification of the one-sided bulge, which is propagating radially. Fig. 15-18 reveal that the deflection of the tube due to the onset of bending decreases as $\lambda_{z}$ increases. Furthermore, for sufficiently large values of $\lambda_{z}$ the tube does not deflect and an axisymmetric bulge develops.

ing to the onset of bulging as well as the formation of the one-sided bulge. Indeed, the deflection of the tube due to the onset of bending decreases as $\lambda_{z}$ increases. It follows that for sufficiently large values of $\lambda_{z}$ the tube does not deflect and an axisymmetric bulge develops.

\section{Conclusions}

A numerical methodology able to capture bifurcation and postbifurcation of residually stressed tubes subjected to a uniform axial loading combined with a uniform internal pressure has been given. The analytical approach to this problem involves a complicated mathematical machinery. Our approach based on the finite element method is an alternative tool to solve it without that complexity. Furthermore, the methodology constructed has been developed in a commercial code widely available to engineering designers, which provides an important source of expansion to engineering analysis and design.

The mechanical modeling of veins has been studied in several previous experimental and biomechanical works in particular in the context of venous tortuosity (see Refs. [26,27]), which in turn is an important instability associated with various pathologies such as varicose veins. Bending bifurcation has been analyzed in that framework, see for instance Ref. [26]. Several factors affect the critical pressure and axial stretch associated with mechanical buckling. Here, the depen- 
dence of bifurcation behavior of tubes under the loading at hand on residual stresses has been illustrated and compared with results when there is no residual stress. Bending and bulging modes are captured. Bulging bifurcation is expected for sufficiently large values of the axial stretch. On the other hand for small values of the axial stretch, the onset of bifurcation is found to be the bending mode. Post-bifurcation is also studied. In the case of bending the structure in subsequent motion after the onset of bifurcation can support higher pressures until bulging is obtained. The bulge, or the abnormal enlargement, appears on one side of the tube showing an irregular shape which is consistent with the development of abdominal aortic aneurysm (AAA). Furthermore, it is shown that bending triggers bulging as opposed to the situation in which bending is not allowed and the onset of bifurcation is associated with bulging. This means that delayed aneurysm formation is possible if bending is not permitted. Nevertheless, in all cases for the material model at hand bulging instability propagates radially which would be associated with an aneurysm rupturing because it cannot sustain more pressure.

A general methodology to deal with residual stresses that are in equilibrium in the absence of external loads has been implemented in a commercial finite element code. Residual stresses have been shown to modify qualitatively the results obtained when there is no residual stress. Therefore, it seems of great importance to consider residual stresses to model arteries and associated cardiovascular diseases such as aneurysms. It is clear that other boundary conditions, as well as other residual stress fields, may give rise to other modes of bifurcation. The developed procedure here can be easily applied to these other conditions.

\section{References}

[1] H. Demirkoparan, J. Merodio, Bulging bifurcation of inflated circular cylinders of doubly fiber-reinforced hyperelastic material under axial loading and swelling, Math. Mech. Solid 22 (2017) 666-682.

[2] J. Rodríguez, J. Merodio, A new derivation of the bifurcation conditions of inflated cylindrical membranes of elastic material under axial loading. Application to aneurysm formation, Mech. Res. Commun. 38 (2011) 203-210.

[3] J. Merodio, D.M. Haughton, Bifurcation of thick-walled cylindrical shells and the mechanical response of arterial tissue affected by Marfan's syndrome, Mech. Res. Commun. 37 (2010) 1-6.

[4] A.A. Alhayani, J.A. Giraldo, J. Rodríguez, J. Merodio, Computational modeling of bulging of inflated cylindrical shells applicable to aneurysm formation and propagation in arterial wall tissue, Finite Elem. Anal. Des. 73 (2013) 20-29.

[5] A.A. Alhayani, J. Rodríguez, J. Merodio, Numerical analysis of neck and bulge propagation in anisotropic cylinders subject to axial loading and internal pressure, Finite Elem. Anal. Des. 90 (2014) 11-19.

[6] A.A. Alhayani, J. Rodríguez, J. Merodio, Competition between radial expansion and axial propagation in bulging of inflated cylinders with application to aneurysms propagation in arterial wall tissue, Int. J. Eng. Sci. 85 (2014) 74-89.
[7] Y.B. Fu, S.P. Pearce, K.K. Liu, Post-bifurcation analysis of a thin-walled hyperelastic tube under inflation, Int. J. Non-Linear Mech. 43 (2008) 697-706.

[8] Y. Fu, G.A. Rogerson, Y.T. Zhang, Initiation of aneurysms as a mechanical bifurcation phenomenon, Int. J. Non-Linear Mech. 47 (2012) 179-184.

[9] Y.B. Fu, Y.X. Xie, Stability of localized bulging in inflated membrane tubes under volume control, Int. J. Eng. Sci. 48 (2010) 1242-1252.

[10] Y.B. Fu, Y.X. Xie, Effects of imperfections on localized bulging in inflated membrane tubes, Phil. Trans. R. Soc. A. 370 (2012) 1896-1911.

[11] Y.B. Fu, J.L. Liu, G.S. Francisco, Localized bulging in an inflated cylindrical tube of arbitrary thickness - the effect of bending stiffness, J. Mech. Phys. Solids 90 (2016) 45-60.

[12] J. Rodríguez, J. Merodio, Helical buckling and post-buckling of pre-stressed cylindrical tubes under finite torsion, Finite Elem. Anal. Des. 112 (2016) 1-10.

[13] J. Merodio, R.W. Ogden, Extension, inflation and torsion of a residually-stressed cylindrical tube, Continuum Mech. Therm. 28 (2016) 157-174 2016.

[14] N.T. Nam, J. Merodio, R.W. Ogden, P.C. Vinh, The effect of initial stress on the propagation of surface waves in a layered half-space, Int. J. Solids Struct. 88 (2016) 88-100.

[15] Hai-Chao Han, K.W. Jennifer, Chesnutt1, Justin R. Garcia, Qin Liu, Wen Qi, Ann. Biomed. Eng. 41 (7) (2013 July) 1399-1410, https://doi.org/10.1007/s10439012-0707-0.

[16] B.L. Langille, Arterial remodeling: relation to hemodynamics, Can. J. Physiol Pharmacol. 74 (7) (1996) 834-841 [PubMed: 8946070].

[17] S. Lehoux, A. Tedgui, Cellular mechanics and gene expression in blood vessels, J. Biomech. 36 (5) (2003) 631-643 [PubMed: 12694993].

[18] S.S. Hayreh, G.E. Servais, P.S. Virdi, Retinal arteriolar changes in malignant arterial hypertension, Ophthalmologica 198 (4) (1989) 178-196 [PubMed: 2748097].

[19] N.C. Taarnhoj, I.C. Munch, B. Sander, L. Kessel, J.L. Hougaard, K. Kyvik, T.I. Sorensen, M. Larsen, Straight versus tortuous retinal arteries in relation to blood pressure and genetics, Br. J. Ophthalmol. 92 (8) (2008) 1055-1060 [PubMed: 18653600].

[20] Z.S. Jackson, D. Dajnowiec, A.I. Gotlieb, B.L. Langille, Partial off-loading of longitudinal tension induces arterial tortuosity, Arterioscler. Thromb. Vasc. Biol. 25 (5) (2005) 957-962 [PubMed: 15746437].

[21] A. Hoger, On the residual stress possible in an elastic body with material symmetry, Arch. Ration. Mech. Anal. 88 (1985) 271-290.

[22] T. Ahamed, L. Dorfmann, R.W. Ogden, Modelling of residually stressed materials with application to AAA, J. Mech. Behav. Biomed. Mater. 61 (2016) 221-234.

[23] P.W. Alford, A.W. Feinberg, S.P. Sheehy, K.K. Parker, Biohybrid thin films for measuring contractility in engineered cardiovascular muscle, Biomaterials 31 (2010) 3613-3621.

[24] M.A. Crisfield, An arc-length method including line searches and accelerations, Int. J. Numer. Methods Eng. 19 (1983) 1269-1289.

[25] SIMULIA, Abaqus Analysis User's Guide, Version 6.14, 2014.

[26] Ricky Martinez, Cesar A. Fierro, Paula K. Shireman, Hai-Chao Han, Mechanical buckling of veins under internal pressure, Ann. Biomed. Eng. 38 (4) (2010 Apr) 1345-1353, https://doi.org/10.1007/s10439-010-9929-1. Published online 2010 Jan 22.

[27] Hai-Chao Han, A biomechanical model of artery buckling, J. Biomech. 40 (16) (2007) 3672-3678, https://doi.org/10.1016/j.jbiomech.2007.06.018. Published online 2007 Aug 8.

[28] Aleksandra Fortier, Vikranth Gullapalli, Reza A. Mirshams, Review of biomechanical studies of arteries and their effect on stent performance, IJC Heart Vessels 4 (2014) 12-18. 